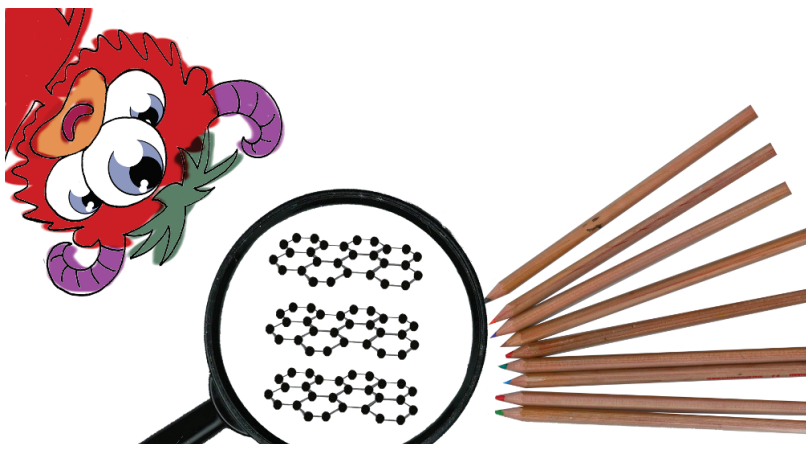

Figure 1. The Nosy Monster and the Carbon Stars - Graphite

Keywords: Crytallography for children, outreach, carbon crystals

\section{MS48-P3 Growing crystals - lighting up dreams - crystal growing competition in Vietnam}

Ngan Nguyen Bich ${ }^{1}$, Luc Van Meervelt ${ }^{2}$

1. Chemistry Department, Hanoi National University of Education (HNUE), Vietnam

2. Chemistry Department, KU Leuven, Belgium

email: ngannb@hnue.edu.vn

In the framework of the project "A joint structural research on platinum(II) complexes for antitumor activity and elaboration of the framework for training in crystallography", funded by VLIR-UOS in Belgium, single crystal crystallography has been introduced at the Chemistry Department of HNUE in Vietnam. On the other hand, the first edition of a Crystal Growing Competition was organized in Vietnam in 2014. When announced many participants, varying from undergraduate students to high school students were very motivated to grow their own crystals.

Initially, students from a few high schools in Hanoi and the Chemistry Department (HNUE) were selected to participate at this first edition. However, members of some Scientific Clubs also asked to attend the competition, and finally more than 100 student groups were registered. Packages with starting material and description of the procedure how to grow a single crystal were delivered to each group. Alum and copper sulfate were used as materials for crystallization.

The final results were evaluated by the organizers as very positive (Figure 1). An official prize awarding ceremony took place in December 2014. Also a video showing the process of the crystal growth was submitted by one of the participating groups illustrating their motivation and enthusiasm.

A small poll among participants illustrated that the students appreciated the new skills they acquired during the competition and showed the need for a next edition. One of the participants has written a news item at the website of her high school and referred to the competition as "Growing Crystals - Lighting up Dreams". This turns out to be the slogan of the 2015 edition of the Crystal Growing Competition in Vietnam, which is now integrated in the IUCr Crystal Growing Competition 2015.

Acknowledgement: $\mathrm{We}$ would like to thank VLIR-UOS (project ZEIN2014Z182), IUCr, KU Leuven and HNUE for supporting the Crystal Growing Competition in Vietnam. 


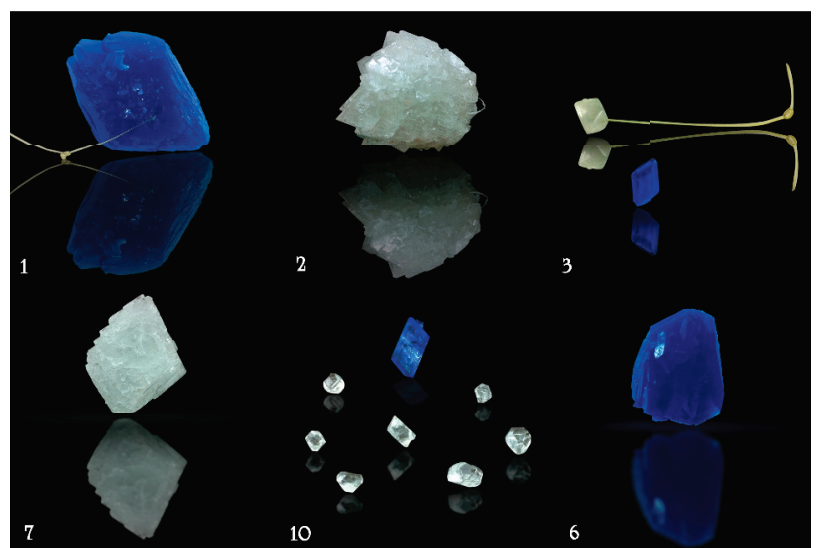

Figure 1. Selected crystals of alum and copper sulfate submitted to the Crystal Growing Competition in Vietnam, edition 2014

Keywords: Crystal growing competition, Vietnam.
MS50. Cultural and historical aspects of crystallography

Chairs: Aleksandar Višnjevac, Petr Bezdička

\section{MS50-P1 Naturally irradiated fluorite as a historic violet pigment: X-ray diffraction and Raman spectroscopic study \\ Petr Bezdička ${ }^{1,2}$, Zdeňka Čermáková, ${ }^{1,2}$, Ivan Němec ${ }^{3}$, Janka Hradilová $^{1}$, David Hradil ${ }^{1,2}$}

1. Academy of Fine Arts in Prague, ALMA laboratory, U Akademie 4, 17022 Prague 7, Czech Republic

2. Institute of Inorganic Chemistry of the ASCR, v.v.i., ALMA laboratory, 25068 Husinec - Řež, Czech Republic

3. Department of Inorganic Chemistry, Faculty of Science, Charles University in Prague, Hlavova 8, 12800 Prague 2, Czech Republic

email: petrb@iic.cas.cz

Naturally irradiated violet fluorite, a cubic $\mathrm{CaF}_{2}$ mineral, is a rare historic pigment. Its documented usage in Europe stretches from ca. 1450 to ca. 1550. The intensely coloured violetish black naturally irradiated fluorite is commonly called antozonite, which is only vaguely defined based on its dark colour and specific odour emanated during grinding. In the published literature, there have been some discrepancies about its physico - chemical properties. Therefore, sixteen samples of antozonite were analysed by X-ray powder diffraction in transmission mode and Raman (micro-) spectroscopy using five different excitation laser wavelengths (445, 532, 633, 780 and $1064 \mathrm{~nm}$ ).

The structural damage of antozonite samples has been assessed by X-ray diffraction and related to their lightness using analysis of image histograms. The XRD study confirmed that the structural disorder caused by natural irradiation may be expressed by broadening of its diffraction lines. The higher is their full-width half-maxima, the lower is the sample's lightness. On the other hand, the reported increase of antozonite's unit cell parameter (and the unit cell volume) is not so straightforward. The studied antozonite samples did not exhibit positive correlation of the parameter and the observed increase of the FWHMs. Still, the sample with the highest full-width half-maxima had also one of the highest values of the unit cell parameter.

Raman spectroscopy revealed specific bands located below $500 \mathrm{~cm}^{-1}$ probably related to radiation-caused defects. Their intensity increased with increasing violet colour saturation, thus providing a specification for antozonite's definition. Spectra excited at 445 and 780 $\mathrm{nm}$ contained also numerous broad bands above $500 \mathrm{~cm}^{-1}$, which seem to be caused by the presence of rare earth elements.

The obtained results have been applied in the analysis of micro-samples of a Late Gothic altarpiece located in an Italian Court in UNESCO city Kutná Hora, Czech 